MS31 Crystal energy

landscapes: computation and

uses

Chairs: Anthony Reilly, Marcus Neumann

\section{MS31-01 Computed Crystal Energy Landscapes: A First Step towards Digital Drug Product Design?}

Susan M. Reutzel-Edens ${ }^{1}$

\section{Lilly Research Laboratories}

email: reutzel-edens_susan_m@lilly.com

Drug product design generally begins with identifying, oftentimes through crystallization screening, solid forms in which to isolate and store the drug substance. When performed under different conditions, crystallization can yield different forms (polymorphs, solvates) with different sizes and morphologies, thus providing an opportunity to engineer particles to desired specifications. To meet the design requirements for the drug product, a solid form must be selected from potentially numerous options discovered through crystallization screening. However, the path to even one commercially-viable form can, in some cases, be lengthy and difficult. Under immense pressure to shorten development timelines and reduce costs, the pharmaceutical industry is keen on right-sizing the time and effort spent on finding suitable solid forms. By contrast, faced with the potential for a product failure should a new, more stable (less soluble) crystal form suddenly appear in a marketed product, finding the most stable crystal form is non-negotiable. In this presentation, the challenges in experimental solid form screening are discussed and the use of computed crystal energy landscapes for enhancing the effectiveness of experimental solid form screens is explored.

Keywords: crystal structure prediction, polymorph, solid form screening, pharmaceutical

\section{MS31-O2 Accurate and Affordable Lattice Energy Calculations: A Solved Problem? \\ Alexandre Tkatchenko ${ }^{1,2}$}

1. University of Luxembourg

2. Fritz-Haber-Institut der Max-Planck-Gesellschaft, Berlin, Germany

email: tkatchen@fhi-berlin.mpg.de

The 6th blind test of crystal structure prediction organized by the Cambridge Crystallographic Data Centre (CCDC) has highlighted the growing maturity of lattice energy calculations based on first-principles density-functional approaches when applied to real-world molecular crystals. Despite clear and significant progress, the existing first-principles calculations are still facing considerable challenges for predicting lattice energies of polymorphic molecular crystals.

In this context, I will summarize the widely used first-principles methods and their successes and failures when applied to the calculation of lattice energies. A particular attention will be paid to the recent exciting finding that many-body dispersion (MBD) effects can play a crucial role in the relative stabilities of molecular crystals of pharmaceutical interest [1]. These findings provide novel control mechanisms in the development and design of intricate polymorphic forms of molecular crystals.

[1] Chem. Sci. 6, 3289 (2015); Angew. Chem. Int. Ed. 52, 6629 (2013); Phys. Rev. Lett. 113, 055701 (2014); Proc. Natl. Acad. Sci. 109, 14791 (2012); Science 351, 1171 (2016).

Keywords: Lattice energies, polymorphs, density functional theory, electronic structure, dispersion 